\title{
Geleitwort des Rektors der Universität Innsbruck
}

\author{
Tilmann Märk
}

Die wechselvolle Geschichte der Innsbrucker Politikwissenschaft ist ein Musterbeispiel für die Ausdifferenzierung der Wissenschaftsdisziplinen in den letzten Jahrzehnten. Die Berufung von Anton Pelinka im Jahr 1975 - damals noch an der Fakultät für Rechtswissenschaften - kann dabei durchaus als "Geburtsstunde“ gesehen werden. Der Etablierung dieser Professur in Innsbruck folgte rasch die Emanzipierung, zuerst 1976 durch die Zuordnung der Professur an die Fakultät für Sozial- und Wirtschaftswissenschaften und gleich im Jahr darauf die Institutsgründung, die wir im vergangenen Jahr zum vierzigsten Mal gefeiert haben. Dabei hatte es das Institut gerade in der "Adoleszenz" nicht immer einfach, war es doch von einem organisatorischen Spezifikum geprägt: Die Studienangelegenheiten waren, entgegen dem Personal, nicht der Sozial- und Wirtschaftswissenschaftlichen Fakultät, sondern den Geisteswissenschaften zugeordnet. Diese Zwitterstellung zwischen den Disziplinen prägten lange Jahre Selbstverständnis und -behauptung des Instituts im inneruniversitären Kontext.

Mit dem sich abzeichnenden Beitritt Österreichs zur Europäischen Union begann für das Institut Ende der 1980er Jahre dann eine neue Phase des Wirkens. Sowohl in fachlicher als auch personeller Hinsicht, insbesondere durch die Berufung von Heinrich Neisser, erfolgte eine Erweiterung des Handlungsspektrums, das bis heute ein konstitutives Element darstellt. Diese neuen inhaltlichen Perspektiven auf europäischer Ebene fanden zudem ihren Niederschlag im regionalen Forschungs- und Lehrumfeld. Kooperationen und Projekte mit den Partner/innen südlich des Brenners, insbesondere im Rahmen der EUREGIO, sind ein wesentliches Standbein des Instituts. Aber auch weitere Berufungen, etwa im Bereich Politische Theorie oder Frauenforschung, unterstützten die Entwicklung und Binnendifferenzierung des Faches.

Prägend war für die Politikwissenschaft sicherlich auch das Inkrafttreten des UG 2002, welches eine Neuordnung der Fakultäten nach sich zog. 
Mit der Gründung der Fakultät für Politikwissenschaft und Soziologie im Jahr 2004 wurden die Sichtbarkeit des Faches sowie die organisatorische Kohärenz deutlich erhöht, wenn auch die Fakultät nach wie vor zu einer der kleinsten der Universität zählt. Die vor kurzem erfolgte Umbenennung in Fakultät für Soziale und Politische Wissenschaften, die Etablierung eines Instituts für Medien, Gesellschaft und Kommunikation sowie die (noch vollständig zu erfolgende) Integrierung des Arbeitsbereichs Peace Studies zeigen, in welch dynamischem Umfeld sich das Institut bewegt. Das spiegelt sich auch in zahlreichen inneruniversitären Forschungskooperationen wider, in denen die Politikwissenschaft vielfältige Anschlussmöglichkeiten zu Nachbardisziplinen bietet.

Diese inhaltliche Erweiterung bringt jedoch auch die Erfordernis, kontinuierlich Überlegungen hinsichtlich der Studienarchitektur anzustellen, um die Politikwissenschaft sowohl in Breite als auch Tiefe adäquat zu vermitteln und attraktiv zu halten. Dass das Institut im Hinblick auf Öffentlichkeitswirksamkeit ein gutes Gespür hat, wurde in der Vergangenheit mehrfach bewiesen - Stichwort „Politiker in residence“ oder die Gastprofessur von Bundespräsident a.D. Heinz Fischer. Dem Institut ist daher für die vergangenen 40 Jahre ganz herzlich zu gratulieren und für die $\mathrm{Zu}$ kunft nur das Beste zu wünschen, um die Strahlkraft der Politikwissenschaft in Forschung, Lehre und "third mission“ zu erhalten bzw. auszubauen und damit die Universität Innsbruck in ihrer Vielfalt maßgeblich zu bereichern!

Univ.-Prof. Dr.Dr.h.c.mult. Tilmann Märk Rektor der Leopold-Franzens-Universität Innsbruck 